\title{
输电线路运行故障的分析与防治
}

郑龙

国网灵宝市供电公司，河南 三门峡 472500

[摘要] 随着市场经济的高速发展和不断完善, 整个社会中的各个行业的竞争是越来越激烈的, 只有在激烈的行业竞争中保持 发展优势, 才可以实现企业的长期稳定、可持续的发展。电力能源企业作为满足人们生产、生活的能源需求的重要保证, 应 在当前的社会发展阶段, 不断的加强电力能源供应的稳定性和共赢之路, 保证电力系统输电线路和相关设备原件的运行安全, 为电力系统的现代化、自动化和智能化提供更多支持。

[关键词]电力系统; 电力输电线路; 运行维护

DOI: $10.33142 /$ hst.v3i1.1369

中图分类号: TM755

文献标识码：A

\section{Analysis and Prevention of Transmission Line Operation Failures} ZHENG Long

State Grid Lingbao Power Supply Company, Sanmenxia, Henan, 472500, China

\begin{abstract}
With the rapid development and continuous improvement of the market economy, competition in various industries in the entire society is becoming increasingly fierce. Only by maintaining the development advantage in the fierce industry competition can the long-term stable and sustainable development of the enterprise be achieved. As an important guarantee for meeting the energy needs of people's production and life, electric energy enterprises should continuously strengthen the stability of electric energy supply and a win-win road in the current stage of social development, and ensure the operation safety of power transmission lines and related equipment components, and provide more support for the modernization, automation and intelligence of power system.
\end{abstract}

Keywords: power system; power transmission line; operation and maintenance

\section{引言}

在当前的电力行业的发展要求下, 国家在电力系统的建设上逐渐增大政策和资金的支持力度, 电力行业的高速发 展和电力基础设施的建设使得输电线路的电压水平呈现出极大的差异化, 电力基础设施基本覆盖了祖国的绝大部分区 域。由于电力系统的输电线路都是暴露在户外的, 会受到各种自然因素和人为因素的影响, 而电力系统的输电线路自 身也会因为长期的负荷使用而出现一定的老化问题。一旦电力系统的输电线路因为自身故障问题或者天气情况、人为 因素的破坏, 会导致大规模的断电问题, 这不仅对整个电力输电系统的部件正常稳定运行产生了严重影响, 也会影响 到人们的生活、工作和生产。

\section{1 输电线路的基本概况}

输电线路这一理论已经被很多人所熟知。但现在电动交通技术发展非常快. 因为运输电力技术推广, 我国输电线 路的布局和设计环节存在很多问题, 在最初的电力基础设施建设过程当中, 由于社会发展也处于比较初级的阶段, 人 们的生活和生产对于电力系统的要求也是比较低的, 所以建造电力系统的相关技术难度也比较小, 对电力系统的输电 线的规格和质量也就比较低。然而, 随着当前的经济社会的高速发展, 人们的生活和生产对电力行业的要求越来越高, 供电质量、供电稳定性的需要与日俱增, 这也推动了电力相关技术的迅速发展, 传统的、简单的电力系统输电线的布 线技术的发展和完善并没有跟上当前这种社会对电力系统的需要。同时, 因为电力系统输电线的布线环节, 没有进行 严格的监督和管理, 使用环节也没有定期的检修和维护, 很容易造成输电线的运行故障。随着电力行业相关技术的发 展、新材料、新工艺、新设备的普及和应用, 木点的电力行业也出现了许多新型的输电线路, 但是, 不可否认的是, 我国目前的电力行业输电线路的整体质量和水平和其他技术先进、电力行业高度的国家相比还有很大的差距。通过对 输电线路运行中存在的故障问题进行不断的分析 ${ }^{[1]}$ 。

\section{2 电力输电线路发生问题的原因}

\section{1 设计不合理}

科学合理、高效高质的设计电力系统的输电线路, 是整个电力系统输电线路在投入使用后可以长期稳定的正常运行的先 
决条件。由于受到了传统的输电线路的施工理念的影响, 当前我国的电力系统的输电线路在设计和规划上还存在一些非常明 显的问题, 这些问题集中体现在设计方案的不科学不合理, 没有考虑到电力行业发展的未来整体趋势和必然要求。

\section{2 施工不规范}

电力系统的输电线路的建造施工的环节, 存在着一定的施工问题, 施工工序和施工标准没有严格的执行, 这主要 是因为输电线路的建造施工的技术人员的专业技术水平和综合素质不符合当前电力行业发展的要求, 工程施工单位的 监督管理工作也没有做到位, 导致了施工环节的质量和标准没有达成, 直接导致了电力系统的稳定、安全运行 ${ }^{[2]}$ 。

\section{3 输电线路常见故障}

\section{1 输电线路自身因素}

一般情况下, 输电线路是由金具、杆塔、导地线和绝缘子等多零部件构成。而构成输电线路的每一个零部件的质 量将决定着输电线路能否安全正常运行。如, 构成输电线路的杆塔常年暴露在自然环境中, 不可避免地受到雨雪和大 风的侵蚀, 如果杆塔的质量不符合要求, 必然会缩短杆塔的使用寿命 ${ }^{[3]}$ 。

\section{2 线路污闪故障}

虽然目前我国的电力行业已经实现了一定的现代化、自动化和智能化, 但是在电力输电线路上还无法完全实现线 路污损问题的发现和处理, 当电力系统的输电线路在运行过程中, 线路表面出现了一定的污染附着, 这会直接导致污 闪的问题出现, 导致电路短路, 跳闸, 直接影响了电力能源的稳定供应, 甚至会损坏电力设备的相关元件。

\section{3 风力破坏}

由于我国地质环境较为复杂的因素导致供电站不得不设置在自然环境比较恶劣的地方, 很容易出现大风破坏输电 线路的情况。由于人们环保意识的不足导致植被被砍伐, 进一步加重了输电线路被大风破坏的情况。例如刮断线路、输 电杆倒塌等。输电线路遭受大风严重破坏不仅使人们生命财产蒙受损失, 而且进一步加重了供电站输送线路的难度。总 而言之, 输电线路遭受严重破坏的关键因素是大风降低了空气中的间隙放电电压。

\section{4 雷击故障}

由于电力系统的输电线路一般都是架设在高空, 特别是一些高压输电线路, 在一些空旷的地方, 输电线路自身架 设的高度又高, 在雷暴天气的时候非常容易收到雷击, 引发系统故障, 甚至直接损坏电力设备 ${ }^{[4]}$ 。

\section{5 覆冰故障}

输电线路运行时另外一种常见故障为覆冰故障, 近年来不断出现极端恶劣天气情况影响了电力输电线路稳定运行, 如果不能有效控制覆冰跳闸问题, 会导致输电线路出现负荷超载的问题, 从而促使输电线路由于不能承受过大荷载而出 现断裂或者变形, 以至于导致严重损坏电线杆绝缘层, 提高输电线路出现事故的概率 ${ }^{[5]}$ 。

\section{6 风偏放电故障}

输电线路运行的时候, 不仅会出现覆冰故障和雷击故障, 而且还存在风偏放电故障, 近年来因为国内大风等恶劣天 气的增多, 导致普遍出现风偏放电故障跳闸现象, 这种问题会严重阻碍电力系统正常运行。如果系统正常运行输电线路 的时候遭受大风天气, 输电线路随风摆动, 将导致线路出现跳闸或者短路的问题。

\section{7 乌害}

鸟类对于电力系统输电线的损伤主要是鸟类在电线上方排便, 鸟粪掉落在输电线上, 会造成电路的短路现象。同 时, 因为输电线路的高度大多和鸟类比较习惯停靠的树木高度差不多, 大量的鸟类也会在输电线路上停留, 这个问题 可能发生在输电线路的所有路段，基本上预防控制起来的难度是非常大的。

\section{4 输电线路运行故障的防治}

\section{1 降低气候等外界因素对输电线路运行的影响}

在当前的电力行业的发展阶段, 大部分电力系统的输电线路都是安装在户外进行架空。换言之, 输电线路所在的 外部环境、自然环境对电力系统的输电线路的稳定、安全运行有很大的影响。同时, 大风、大雨、雷暴、冰雹这些气 候环境的因素具有非常强的不可抗力, 变化也非常难以预测。为此, 电力行业的输电线路维护管理人员只能尽量的做 到对气候环境进行监测, 及时的对那些比较确切的自然气候灾害做出提前应对 ${ }^{[6]}$ 。

\section{2 防治外力破坏措施}

4.2. 1 提高输电线路施工人员素质, 增加传输线巡逻人员数量

在电力系统的输电线路的运行过程中, 施工和管理人员也是其中影响输电线路质量和稳定性的一个重要因素, 因 
此, 在对人员的选择方面应该提高标准, 此外, 电力系统的管理人员还应为输电线路的建造施工人员提供有效的职业 技能培训, 以有效的提高这些一线施工人员的输电线路的相关专业能力, 以避免在线路的建设、维护过程中, 因为自 身的专业技术能力和养护经验的不足, 而造成输电线路的运行故障和安全问题。同时, 需要积极地引进高素质、高水 平、高技术能力的专业技术人员, 保证他们可以定期的对输电线路进行检查和监测, 保证输电线路和相关设备的正常 运行，及时消除线路当中的故障和问题。

4.2. 2 安装防鸟刺和防鸟帽

在电力系统的输电线路上, 为了有效的防止鸟类在输电线路上停留, 可以安装一定的防鸟刺, 防止由于鸟类分辨 的影响是的输电线路出现短路故障的问题。

\section{5 构建出输电线路一体化运维体系}

在监控系统中，建立了故障分析系统和维护运行系统，同时还建立了集成运维系统。感知和监控联机状态，逐渐 形成一个地对空三维系统. 无人驾驶飞机、直升机、检测系统组成的一个机器人, 或各种类型的在线监测设备的监控 系统等等。

\section{6 结束语}

当前社会, 人们的生活生产对于电力系统的要求越来越高, 电力行业不仅要提供稳定高质量的电源供应, 也要有 力的保证电力能源相关设备和输电线路的运行安全。

\section{[参考文献]}

[1]张倩.输电线路运行故障的分析与防治分析 $[\mathrm{J}]$. 花炮科技与市场, 2018(04): 94 .

[2] 纪云嵘. 输电线路运行故障因素分析及防治措施探讨 $[\mathrm{J}]$. 电子世界, 2017(15) : 75 .

[3] 张贺林. 输电线路运行故障的分析与防治 $[\mathrm{J}]$. 黑龙江科技信息, 2017 (18): 90 .

[4]曹诗冰.输电线路运行故障分析与防治对策 [J].中国高新技术企业,2016 (29) : 120-121.

[5]孙竞明.输电线路运行故障的分析与防治 $[\mathrm{J}]$. 科技与创新, 2016 (04) : 136-140.

[6]彭玉金,宁琦. 试分析输电线路运行故障分析与防治策略 [J].信息化建设, 2016 (02): 289.

作者简介: 郑龙 (1983.12-), 男, 毕业于华北电力大学, 专业电气工程及其自动化, 现就职于国网灵宝市供电公司, 职务主任，高级工程师。 